\title{
Strongly Coupled Ionic Mixtures and the H/He EOS
}

\author{
Hugh E. DeWitt \\ Lawrence Livermore National Laboratory, Livermore, CA 94550
}

\begin{abstract}
This paper summarizes recent work on the strongly coupled OCP and Binary Ionic Mixture equation of state and other thermodynamic quantities in white dwarf interior conditions for both fluid and solid phases with the assumption of a uniform background. Conditions for phase separation of different elements in fluid or solid phases is strongly dependent on deviations from the linear mixing rule which gives the equation of state as an additive function of the OCP equation of state. These deviations turn out to be small (a few parts in $10^{5}$ ) and always positive including the case where the fraction of the higher $\mathrm{Z}$ component approaches 0 . Also the equation of state of strongly coupled light elements ( $\mathrm{H}$ and $\mathrm{He}$ particularly) obtained from simulations with a linear response description of the electrons is given for conditions appropriate to brown dwarf star interiors. Recent Livermore work on a band structure calculation of the enthalpy of $\mathrm{H}$ and $\mathrm{He}$ mixtures under jovian conditions is discussed. This work leads to a prediction of a high temperature $(15000 \mathrm{~K})$ for miscibility of He in ionized $\mathrm{H}$ at $10 \mathrm{Mb}$.
\end{abstract}

\section{Resume}

Ce papier resume l'ouvrage recent sur le OCP à fort couplage et sur l'equation d'état et d'autres quantites thermodynamiques pour le melange binarire ionique aux conditions interieure des nains blancs. Les conditions pour seperation de phae dan les éléments divers dans l'état solide ou fluide sont très sensible aux deviations de regle lineaire qui donne l'equation d'état comme function additive sur celui du OCP. Cettes deviations sont très petities (quelques parts dans $10^{5}$ ) et toujours positive meme guard la fraction du composant a $Z$ superieur va vers $O$. L'equation d'état pour les èlèments lèger (surtout $\mathrm{H}$ et $\mathrm{He}$ ) a fort couplage obtenu par simulations avec response lineaire des electrons est donné p;our les conditions des nains brun. Nous discutons aussi calculs recent, fait à Livermore, prèdisent que le He dans $\mathrm{H}$ est immiscible a haut temperature $(15000 \mathrm{~K})$ à la pression de $10 \mathrm{Mb}$. 


\subsection{Introduction}

Hydrogen and helium mixtures form the main components of jovian planets, brown dwarf stars, and ordinary main sequence stars. In these various conditions the state of the mixtures ranges from molecular and atomic fluids in the outer layers of the jovian planets, to ionized $\mathrm{H}$ and neutral He deeper in jovian planets, to fully ionized low $\mathbf{Z}$ elements in brown dwarfs and main sequence stars. Hydrogen and helium are found on the surfaces of white dwarf stars but most of the interiors are composed of heavier elements beginning with $\mathrm{C}, \mathrm{O}, \mathrm{Mg}$, and up to $\mathrm{Fe}$ that are fully ionized. The relativistically degenerate electron gas provides a nearly uniform density neutralizing background and the positive pressure that balances the gravitational contraction force and thus determines the size of the white dwarf. For applications to these various astrophysical objects we need to know the equation of state of mixtures of light elements from the molecular-atomic region at low temperature and high density on up to the extreme high densities where the ions are bare nuclei. One can distinguish four density regions:

i) Ionic fluid in a (nearly) uniform background of degenerate electrons, $\mathbf{r}_{\mathbf{s}} \rightarrow 0$. An example is the $\mathrm{C}$ and $\mathrm{O}$ fluid (bare nuclei, usually assumed to be classical) above the crystallizing core of a white dwarf star ${ }^{1}$. Monte Carlo simulations give very accurate results for the ionic interactions in both the fluid and the solid state. This system is well understood classically, though quantum effects are now known to play a strong role in real white dwarfs 2 .

ii) Ions in a responding background of degenerate electrons ( $\left.r_{s}<1\right)$. This is the situation in brown dwarf star interiors with pressures above $80 \mathrm{Mb}$ so that both $\mathrm{H}$ and $\mathrm{He}$ are fully ionized. The equation of state is given with fairly good accuracy by using Monte Carlo simulations with the Coulomb potential screened by using an appropriate dielectric function in linear response theory. Also fairly accurate results can be obtained by solving coupled Hypernetted Chain (HNC) equations for the ionic mixtures ${ }^{3}$. At the moderate temperature ( $\mathrm{a}$ few $\mathrm{eV}$ ) but density high enough that $0.1<\mathrm{r}_{\mathrm{S}}$ $<1)$ the electrons are highly degenerate $\left(k T<\mathrm{E}_{\mathrm{F}}\right)$ but still polarizable.

iii) Partially Ionized Mixtures $\left(r_{s} \gtrsim\right)$ with the ions still strongly coupled. For $\mathrm{P} \geq 3 \mathrm{Mb}$ as in the interior of most of Jupiter one finds $\mathrm{H}^{+}$along with neutral $\mathrm{He}$, and consequently both free and bound electrons. In this density and temperature region both $\mathrm{MC}$ and $\mathrm{HNC}$ calculations are of questionable 
accuracy, and there is still considerable uncertainty. A plasma phase transition ${ }^{4}$ is possible for the hydrogen at roughly $\mathrm{kT} \approx 1 \mathrm{eV}$. Such a transition may be modified by the presence of neutral helium $5,6,7$. This region is unfortunately still outside the possible region of experimental measurements.

iv) Neutral $\mathrm{H}_{2}$ and $\mathrm{He}$ as in jovian atmospheres, $0<\mathrm{P}<3 \mathrm{Mb}$. This region has been studied experimentally to the $1 \mathrm{Mb}$ region for $\mathrm{H}_{2}$ and is accessible to $3 \mathrm{Mb}$ in future experiments.

Most of this paper will deal with region i), the extremely high density region where one has some hope of obtaining some nearly exact results from very long numerical simulations, either Monte Carlo or molecular dynamics. Yakovlev and Shalybkov ${ }^{1}$ have given an excellent review of most known results up until 1988 for the OCP and ionic mixtures in the strong coupling region, and discussed the applications to very dense stars, particularly white dwarf interiors. Problems that remain to be addressed with greater quantitative precision include the questions of the value of the coupling parameter at which the fluid solid transition occurs, and the possibility of phase separation, i.e. separation of heavy from light elements in the freezing process or even in the fluid region. Associated with the phase separation process is the need for an accurate representation of the equation of state of the ionic mixtures in both fluid and solid phases. The well known linear mixing rule gives an excellent first approximation to the mixture equation of state in terms of the OCP results for the energy and the Helmholtz free energy. Small deviations from the linear mixing rule determine the phase diagram of mixtures, for example the possible separation of $\mathrm{Fe}$ from the $\mathrm{C}$ and $O$ in the fluid region of the white dwarf interior. These questions need to be addressed by the generation of very accurate Monte Carlo energy data for ionic mixtures using several hundred to a thousand particles and averaging over as many as a few hundred million configurations. Some new results for mixtures will be given here.

\subsection{Strongly Coupled Ionic Mixtures in a Uniform Background}

We will use the now standard definitions of parameters. For the OCP with number density $n=N / V$, temperature $\beta=1 / k T$, and classical point charges Ze moving in a uhiform background the coupling parameter is $\Gamma=$ $(\mathrm{Ze})^{2} / \mathrm{akT}$ with $\mathrm{a}=\left(\frac{4 \pi}{n}\right)^{-1 / 3}$, the Wigner-Zeitz or ion sphere radius. At the extreme densities in white dwarf stars the deviation from a uniform 
background due to ionic polarization of the relativistic electrons is measured by $r_{s}=a_{e} / a_{B}$ with $a_{c}=a / Z^{1 / 3}$ the electron sphere radius. $r_{s}$ from about 0.01 for $p=10^{6} \mathrm{gm} / \mathrm{cc}$ down to 0.001 for $\rho=10^{9} \mathrm{gm} / \mathrm{cc}$, so that to a good approximation electron screening effects on the ion-ion contribution to the equation of state is quite small. The interior of a white dwarf if it were a single element is believed to be crystallized with an energy of the form:

$$
\mathrm{U} / \mathrm{NkT}=\left(\mathrm{U}_{0}+\mathrm{U}_{\mathrm{th}}\right) / \mathrm{NkT}=\mathrm{a}_{\mathrm{M}} \Gamma+\left(3 / 2+\mathrm{A}_{1} / \Gamma+\mathrm{A}_{2} / \Gamma^{2}+\ldots\right)
$$

where $\mathrm{aM}_{\mathrm{M}}=-0.89592926$ is the Madelung constant for the bcc lattice, $3 / 2$ is the classical thermal energy of the harmonic lattice, and the first anharmonic energy 8 term has the coefficient $A_{1}=10.84$. The second anharmonic energy term ${ }^{9}$ is known only from $M C$ simulations and is $A_{2}=$ 600. The Helmholtz free energy for the OCP bcc lattice can be obtained by temperature integration:

$$
\mathrm{F} / \mathrm{NkT}=\mathrm{a}_{\mathrm{M}} \Gamma+\frac{3}{2} \ln \Gamma+\mathrm{C}-\mathrm{S}_{\mathrm{H}}-\mathrm{A}_{1} / \Gamma-\frac{\mathrm{A}_{2}}{2 \Gamma^{2}}+
$$

where $C=1+\ln \left(2(3 / 4 \pi)^{1 / 3}\right)$ and $S_{H}$ is the entropy constant $(<\ln \omega\rangle$, averaged over lattice phonon frequencies which for the OCP bcc lattice is 2.4939 .

The OCP fluid energy, U/NkT, obtained from long Monte Carlo simulations ${ }^{10}$ in the strongly coupled region, $1<\Gamma \leq 200$ again has the remarkable property of splitting to a good approximation into a static piece and a thermal piece, but with the thermal energy governed by a power law:

$$
\mathrm{U} / \mathrm{NkT} \equiv\left(\mathrm{U}_{0}(\rho)+\mathrm{U}_{\mathrm{th}}(\rho, \beta)\right) / \mathrm{NkT}=\mathrm{a}_{\mathrm{F}} \Gamma+b \Gamma^{s}+\mathrm{c}
$$

where $s$ is a number ranging from $1 / 4$ to $2 / 5$ depending on the number of terms used in the fitting form in Eq. 3; the best estimate is $s=1 / 3$. Integration over the temperature from $\beta$ to 0 , gives the OCP fluid free energy as;

$$
\mathrm{F} / \mathrm{NkT}=\mathrm{a} \mathrm{F} \Gamma+(1 / \mathrm{s}) \mathrm{b} \Gamma \mathrm{s}+\mathrm{cln} \Gamma+\mathrm{D}
$$

Where $D$ is an integration constant. The coefficient of $\Gamma$ in Eqs 3 and 4 is the 'fluid Madelung coefficient' which Rosenfeld 11 has shown to have an exact limiting value of $\mathrm{a}_{\mathrm{F}}=-9 / 10$, which is the value in the $\Gamma \rightarrow \infty$ for the fluid. If $a_{F}$ is allowed to be a fitting parameter for the OCP fluid data the 
coefficients for the fit to the best available OCP MC data is aF $=-0.89921$, $\mathrm{b}=0.596, \mathrm{c}=0.268$ and $\mathrm{s}=0.3253$. Other equally accurate fits to the OCP fluid data with af fixed at $-9 / 10$ are given in Ref. 10 . Generally the OCP energy data for large $\Gamma$ is known to about \pm 0.0005 (which is a few parts in $10^{6}$ ), and simple fitting functions like Eq. 3 can reproduce the known OCP data to about \pm 0.001 . This kind of accuracy is ultimately needed also for the ionic mixtures in order to determine the freezing line for ionic mixtures and the conditions for phase separation in the freezing process or in the fluid. For the OCP the fluid and solid Helmholtz free energies cross at $\Gamma=172$, which should be regarded as the best available estimate of the OCP freezing transition $\Gamma$. The inclusion of the first order anharmonic energy in Eq. 1 has changed the estimate from the earlier value $^{12}$ of 178 to 172 . Since the fluid and bcc solid free energies have so nearly the same slope, a very small change in one of the free energies can send the crossing point up or down very much.. This fact will be even more true for ionic mixtures which means that current estimates ${ }^{13}$ of the phase diagrams for ionic mixtures must be regarded with some skepticism.

It should also be noted that the classical OCP and the classical ionic mixture is a serious approximation for white dwarf star interiors since in fact the ions in these stars have serious quantum diffraction effects 2 . The measure of QM diffraction effects is $\eta=h \omega_{\mathrm{p}} / \mathrm{kT}$ which ranges from 3 to 8 in white dwarf interiors. However, the location of the freezing transition is only slightly affected by quantum effects. The transition temperature is lowered ( $\Gamma$ increased), but even for $\eta=8$ the transition $\Gamma$ is changed to only abut 200.

Rosenfeld has demonstrated a number of other exact limiting results for the OCP fluid and the ionic mixtures for large $\Gamma$. The screening function, $H(x)$ with $x=r / a$, in the pair correlation function:

$$
\mathbf{g}(\mathbf{x})=\exp \{-\Gamma / x+H(x)\}
$$

is needed for calculations of the screening enhancement of thermonuclear reactions in very dense stellar interiors. $\mathrm{H}(0)$ gives the lowering of the Coulomb barrier for two rapidly approaching ions. The Onsager molecule method used by Rosenfeld gives a result for the screening function for finite $\Gamma$ :

$$
H(x) / \Gamma=h_{0}(\Gamma)-h_{1} x^{2}+h_{2}(\Gamma) x^{4}-h_{3}(\Gamma) x^{6}+
$$


In this expansion around the $x=0$ only the coefficient $h_{1}$ is a constant independent of $\Gamma$, namely $h_{1}=1 / 4 . h_{2}$ and $h_{3}$ must be determined from the best available MC data for $g(x)$. For large $\Gamma(100$ to 200$) h_{2} \approx 0.038$ which is large enough to influence the determination of $h_{0} . H(0)=h_{0} \Gamma$ is given by the difference of the Helmholtz free energy for $N$ charges $Z$ and the free energy of $N-2$ charges $Z$ and on charge of $2 Z$. The result can be shown to be:

$$
\begin{aligned}
\mathrm{H}(0) & =2 \mathrm{f}_{\mathrm{OCP}}(\Gamma)-\mathrm{f}_{\mathrm{OCP}}\left(2^{5 / 3} \Gamma\right)-\left(\partial / \partial \mathrm{x}_{2}\right) \Delta \mathrm{f}_{\mathrm{OCP}}(\Gamma) \\
& =(9 / 10)\left(2^{5 / 3}-2\right)=1.0573 \Gamma \text { as } \Gamma \rightarrow \infty
\end{aligned}
$$

where $x_{2}=N_{2} /\left(N_{1}+N_{2}\right)$. The first line of Eq. 6 is the linear mixing rule result for $H(0)$ with $\Delta f_{O C P}=f_{\text {mixture }}-f_{L M}$, and $f=F / N k T$. Rosenfeld has shown that by inclusion of the $x^{4}$ term in Eq. 5 , that $H(0)$ can be obtained very accurately without using the linear mixing rule ${ }^{14}$, and that the results agree remarkably well with the linear mixing result. The sign of $\Delta f$ is quite important for this discussion.

The general statement of the linear mixing rule for binary ionic mixtures is:

$$
\begin{aligned}
& U\left(Z_{1}, x_{1}, Z_{2}, x_{2}\right) / N k T=-\frac{9}{10}\left\{x_{1} Z_{1}^{5 / 3}+x_{2} Z^{5 / 3}\right\} \Gamma_{e}+U_{t h} / N k T \\
& \approx x_{1} f_{O C P}\left(\Gamma_{1}\right)+x_{2} f_{O C P}\left(\Gamma_{2}\right)
\end{aligned}
$$

with $\Gamma_{1}=\mathrm{Z}_{1}{ }^{5 / 3} \Gamma_{\mathrm{c}}$. Linear mixing applies exactly in the large $\Gamma$ limit but is at best a good approximation for the fluid mixture thermal energy. Thus the usual statement of the linear mixing rule in Eq. 7 is indicated as an approximation. It is the deviations from linear mixing in the binary ionic thermal energy that result in the possibility of phase separation, i.e. $Z_{2}$ ions separating from the $Z_{1}$ ions, and similarly the possible separation of large $Z$ ions from smaller $Z$ ions in the freezing process.

Ogata, et al 13 reported conditions for phase separation of high $\mathrm{Z}$ ions in the fluid phase and phase diagrams for binary ionic mixtures upon freezing which were very much influenced by their MC observations of some negative deviations from linear mixing in the limit of a small fraction of the large $\mathrm{Z}$ component, i.e. $\mathrm{x}_{2} \rightarrow 0$. Our recent results (Slattery and DeWitt ${ }^{15}$ ) indicate that the deviation from linear mixing is only positive. 
Brami, Joli, and Hansen 16 did a detailed study of the conditions for phase separation in binary ionic fluids using the pure HNC mixture equations (no bridge function correction) and found always positive deviations with a magnitude of never more than 0.027 for $x_{2}=0.05$ and $Z_{2} / Z_{2}=8$. Ogata et all 13 reported negative deviations for MC binary fluid runs with $N_{1}=990$, $Z_{1}=1$, and $N_{2}=10, Z_{2}=3$ and 5 for $\Gamma_{1}=20$, thus $x_{2}=0.01$. We first tried the $Z_{2}=3$ case with $\mathrm{HNC}$ mixture equations and found that $\mathrm{U}_{\text {mix }} / \mathrm{NkT}=-17.4614$ and that $\mathrm{U}_{\mathrm{LM}} / \mathrm{NkT}=-17.4621$ (also from $\mathrm{HNC}$ ).

The difference is $\Delta \mathrm{U}_{\mathrm{HNC}} / \mathrm{NkT}=+0.0007$. All mixture runs done with the $\mathrm{HNC}$ equations give positive deviations from linear mixing.

Since the HNC equation is an approximation, the above results may be questioned with the presumably more accurate $\mathrm{MC}$ fluid mixture simulations. We did a few MC mixture runs for $x_{2}=0.01\left(N_{1}=990\right.$ and $\mathrm{N}_{2}=10$ ) but with 150 million configurations which is 20 times the number of configurations reported by Ogata et al. For $\Gamma_{1}=20$ our MC mixture result was $U_{\text {mix }} / \mathrm{NkT}=-17.7231$ and the linear mixing result using the best available fit to the OCP fluid energy data, Eq. 3, is $\mathrm{U}_{\mathrm{LM}} / \mathrm{NkT}=-17.7241$. Thus we obtain a positive deviation of $\Delta \mathrm{U}_{\mathrm{MC}} / \mathrm{NkT}=+0.0010$, whereas Ogata et al report a value of -0.002 . A possible source of error in the Ogata et al results is their fitting function for the OCP data which is inaccurate by about 0.003 for some values of $\Gamma$. The deviations from linear mixing are obviously very small and the reported results depend very much on the accuracy of the MC energy results and the accuracy of the fit to the OCP fluid data. Our conclusion is that the MC mixture results are always positive.

A positive deviation from linear mixing has a number of consequences. Ogata, Iyetomi, and Ichimaru ${ }^{16}$ evaluated $H(0)$ from their MC data for $g(r)$ on the assumption that $h_{2}$ in Eq. 5 was 0 , whereas Rosenfeld 14 finds $h_{2} \approx$ 0.038 in agreement with Alastuey and Jancovici 17 from their work on enhancement of thermonuclear reactions in 1978. The Ogata et al ${ }^{16}$ results for $\mathrm{H}(0) / \Gamma$ are about $2 \%$ larger than the results of Rosenfeld and of Alastuey and Jancovici which are also in close agreement with the linear mixing rule. Ogata et al ${ }^{13}$ (in their report to the Rochester Conference) cite the correction term in Eq. 6 to explain this difference. However, their agreement with linear mixing plus the correction requires that their deviation from linear mixing be negative, but our results indicate that $\Delta \mathrm{f}$ is positive. The $2 \%$ difference in estimates of $h_{o}=H(0) / \Gamma$ makes a factor of 10 difference in the final enhancement rate for thermonuclear reactions at large $\Gamma$. As Isern has pointed out at this Conference ${ }^{18}$ a very small change in the enhancement of thermonuclear reactions in a white dwarf star at the Chandrasekhar limit can make the difference in the star becoming a supernova or to collapse into a 
neutron star. For reasons given above we think the Alastuey-Jancovici estimates ${ }^{17}$ of the screening enhancement of thermonuclear reactions are more accurate and reliable than the results of Ogata, Iyetomi, and Ichimaru 16 .

The crystallization of binary ionic mixtures provides another example for which the sign of the deviation from liner mixing can make a qualitative difference. Using a density functional theory of freezing Segretain and Chabrier ${ }^{19}$ estimated the domains of three types of phase diagrams depending upon the ratio of the charges, $Z_{1} / Z_{2}$. For $0.72<Z_{1} / Z_{2}<1$ the phase diagram for solidification is a spindle type; for $0.58<Z_{1} / Z_{2}<0.72$ an azeotropic phase diagram results; and for an even greater charge disparity, $\mathrm{Z}_{1} / \mathrm{Z}_{2}<0.58$, a eutectic phase diagram results. This is the same progression of phase diagrams as is found for mixtures of hard sphere diameters with different diameters. Ogata et al ${ }^{13}$, however, based on their $\mathrm{MC}$ simulations that lead to negative values of $\Delta \mathrm{f}$, find that the azeotropic form can persist for any value of $Z_{1} / Z_{2}$ near 1 when the fraction of the higher $\mathrm{Z}$ component is very small, i.e. $x_{2} \approx 0.01$. The positive values of $\Delta f$ that we find support the spindle shaped phase diagram for all values of $x_{2}$ for $Z_{1} / Z_{2}$ near 1 . the type of phase diagram for binary ionic mixtures in white dwarf stars, $C$ and $O$, and $C$ and $F e$, has major astrophysical consequences for the possible separation and crystallization of trace elements ${ }^{19}$, and the resulting effect on the cooling time for white dwarfs. It is clear that a lot more very accurate numerical simulation of binary ionic mixture energies is needed to finally settle these questions.

\subsection{Strongly Coupled Ionic Mixtures in a Responding Background}

For applications to brown dwarf stars and the jovian planets the light elements, especially $\mathrm{H}$ and $\mathrm{He}$, may be partially to fully ionized. Although the free electrons are largely degenerate the $r_{S}$ value is typically in the vicinity of 0.5 to 1 . Thus the electrons can screen the protons and alpha particles appreciably. The electrons may also have finite temperature effects measured by the parameter $\theta=\mathrm{kT} / \mathrm{E}_{\mathrm{F}}$ which leads to complete degeneracy when $\theta \ll 1$, but in some stars we may have the worst of all possibilities, namely $\theta \approx 1$. Both the density dependence $\left(r_{s}\right)$ and the temperature dependence $(\theta)$ can be dealt with by using an appropriate density and temperature electron dielectric function in linear response theory. This has been developed by Chabrier and Ashcroft ${ }^{20}$ for solution in the HNC ion mixture equations. The energy and pressure of the ionic mixture in linear response (from their paper) is: 


$$
\begin{aligned}
U^{e x}=\langle E\rangle+ & \beta\left\langle\frac{\partial E}{\partial \beta}\right\rangle_{v} \\
u^{e x}=\frac{\beta U^{e x}}{N}= & \frac{1}{2} \frac{\beta}{(2 \pi)^{3}}\left\langle Z^{2}\right\rangle \int d k v(k)\left[S_{z}(k)-1\right] \\
& +\frac{1}{2} \frac{\beta}{(2 \pi)^{3}}\left\langle Z^{2}\right\rangle \int d k v(k)\left[\frac{1}{\varepsilon(k)}-1\right] S_{z}(k) \\
& +\frac{1}{2} \frac{\beta}{(2 \pi)^{3}}\left\langle Z^{2}\right\rangle \int d k v(k)\left[\frac{\partial}{\partial \beta}\left[\frac{1}{\varepsilon(k)}\right]\right]_{v} \\
P_{z}^{e x}=\frac{1}{3}\langle E\rangle & -\left\langle\frac{\partial E}{\partial \beta}\right\rangle_{\beta}, \\
& -\frac{1}{2} \frac{\beta}{(2 \pi)^{3}}\left\langle Z^{2}\right\rangle \int d k v(k)\left[V \frac{\partial}{\partial V}\left[\frac{1}{\varepsilon(k)}\right]\right]_{\beta} S_{z}(k) \\
& +\frac{1}{6} \frac{\beta}{(2 \pi)^{3}}\left\langle Z^{2}\right\rangle \int d k v(k)\left[\frac{1}{\varepsilon(k)}-1\right] S_{z}(k) \\
p^{e x}=\frac{\beta P^{e x}}{\rho} & =\frac{1}{6} \frac{\beta}{(2 \pi)^{3}}\left\langle Z^{2}\right\rangle \int d k v(k)\left[S_{z}(k)-1\right] \\
& \\
&
\end{aligned}
$$

In Eqs. 8 and $9 \mathrm{E}$ is the total ion-ion and ion-electron interaction energy and pex is the corresponding pressure contribution. $v(k)$ is the Fourier transform of the Coulomb potential, $4 \pi \mathrm{e}^{2} / \mathrm{k}^{2}, \mathrm{~S}_{\mathrm{z}}(\mathrm{k})$ is the structure factor, and $\varepsilon(k)$ is the density and temperature dependent dielectric function. The first term in $u^{c x}$ and $p^{c x}$ is the usual BIM functions appropriate for a uniform background, $r_{s}=0$. The second term is the linear response density contribution, and third term is the result of the temperature dependence in the dielectric function. The screened potential, $v(k) / \varepsilon(k)$, is used in the 
solution of the coupled HNC equations for the BIM in order to calculate $S_{\mathrm{Z}}(\mathrm{k})$ which is then used in Eqs. 8 and 9 to evaluate the energy and pressure of the mixture. Chabrier and Ashcroft presented results for a variety of mixtures, $\mathrm{H}$ and $\mathrm{He}, \mathrm{H}$ and $\mathrm{O}$, with temperatures and densities that went from weak coupling to strong coupling and with $x_{2}=0, .25, .75$, and 1 . They checked the linear mixing rule and found that deviations from linear mixing ranged from $0 \%$ to at most $3 \%$ and were positive. The largest deviations from linear mixing occurred for $\theta=1$, the most difficult temperature region.

For the very high temperatures in the center of the sun Iyetomi and Ichimaru ${ }^{21}$ have solved the $\mathrm{HNC}$ equations for $\mathrm{H}$ and $\mathrm{Fe}$ mixtures in order to construct a phase diagram for $\mathrm{H}$ and $\mathrm{Fe}$. They find that the central temperature of the sun is too hot by a factor three to allow for thepossibility of any separation of Fe from the remaining solar plasma. Their results suggest that heavy elements, such as Fe, might be able to separate from small cool stars with interior temperatures of only a few million ${ }^{\circ} \mathrm{K}$.

Eqs. 8 and 9 could in principle be used for evaluation by Monte Carlo simulation, though this has not been done yet with a temperature dependent dielectric function. Hubbard and DeWitt have done extensive MC calculations on $\mathrm{H}$ and $\mathrm{He}$ mixtures 22 , assumed to be fully ionized, with the density dependent RPA dielectric function (the Lindhard function) and $\theta=$ 0 . $45 \mathrm{MC}$ simulations were done on pure $\mathrm{H}$, pure $\mathrm{He}$, and mixtures of $\mathrm{H}$ He with values of $r_{s}$ ranging from 0.2 up 1.4 and values of $\Gamma_{e}$ ranging from 7 to 155 . Three mixtures were computed: $80 \% \mathrm{H}$ and $20 \% \mathrm{He}, 50 \%$ and $50 \%$, and $20 \%$ and $80 \%$. Unfortunately because of limited computer time the number of particles used was only $\mathrm{N}_{1}+\mathrm{N}_{2}=50$, and the energies were obtained after thermalization with $10^{5}$ configurations. By today's standards these simulations are far too short and too few particles to give accurate results for the energy and pressure. Nevertheless, enough data was obtained that a Helmholtz free energy model could be constructed:

$$
f=F_{\text {mix }} / N k T=-a \Gamma_{c}+b \Gamma_{e}^{1 / 4}-\operatorname{cln} \Gamma_{e}+d
$$

which is similar to Eq. 4 except the exponent $s$ was chosen to be $1 / 4$ and the coefficients in Eq. 10 are all functions of $r_{s}$. Specifically, the coefficient of $\Gamma_{\mathrm{e}}$ was expanded to the second power in $\mathrm{r}_{\mathrm{s}}$ :

$$
\begin{aligned}
& a\left(r_{s}\right)=a_{1}\left[(1-x) Z_{1}^{5 / 3}+x Z_{2}^{5 / 3}\right]+a_{2}(1-x) r_{s}+a_{3} x r_{s} \\
& +\left[a_{4}(1-x)+a_{5} x\right] r_{s}^{2}
\end{aligned}
$$


The coefficients $b, c$, and $d$ were modeled with only the linear dependence in $\mathbf{r}_{\mathbf{s}}$. All together there were 10 numerical coefficients $\left(\mathbf{a}_{1}, \mathbf{a}_{2}, \ldots\right)$ that were obtained numerically by a least squares fit to the $45 \mathrm{MC}$ data points. These coefficients used in Eq. 10 gave a useful numerical model for the free energy mixture of $\mathrm{H}^{+}, \mathrm{He}^{++}$, and the screening electrons. In order to obtain the equilibrium curves for the coexistence of two liquid phases at a pressure of $8 \mathrm{Mb}$, the equilibrium equations were solved:

$$
\begin{aligned}
& \mu_{1}\left(T, N_{1} / V, N_{2} / V\right)=\left(\partial F / \partial N_{1}\right)_{T, V, N}, \\
& \mu_{2}\left(T, N_{1} / V, N_{2} / V\right)=\left(\partial F / \partial N_{2}\right)_{T, V, N}, \\
& \mu_{1}\left(T, N_{1 a} / V, N_{2 a} / V\right)=\mu_{1}\left(T, N_{1 b} / V, N_{2 b} / V\right), \\
& \mu_{2}\left(T, N_{1 a} / V, N_{2 a} / V\right)=\mu_{2}\left(T, N_{1 b} / V, N_{2 b} / V\right), \\
& P\left(T, N_{1 a} / V, N_{2 a} / V\right)=P\left(T, N_{1 b} / V, N_{2 b} / V\right), \\
& P\left(T, N_{1 a} / V, N_{2 a} / V\right)=P_{s},
\end{aligned}
$$

using Eq. 10. The resulting equilibrium curve for the coexistence of to liquid phases is shown in Fig. 1 (from the Hubbard-DeWitt paper)

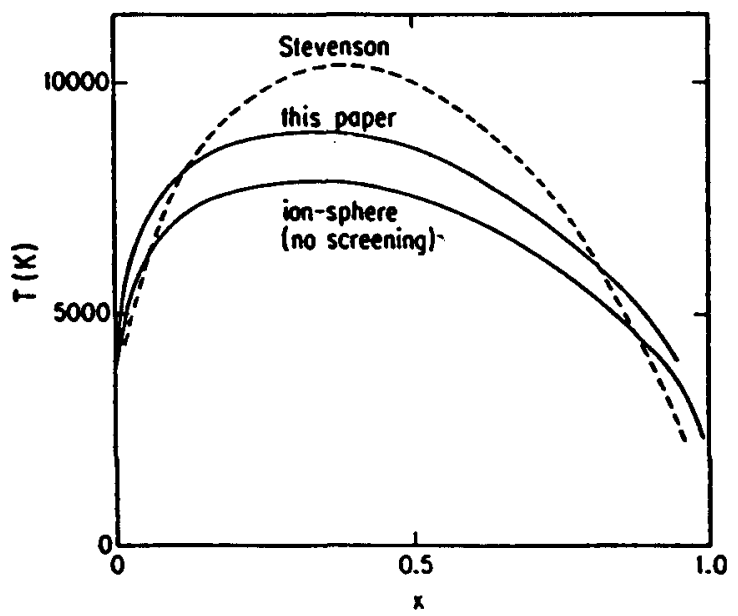

Fig. 1 - Fquilibrium curve for coexistence of two liquid phases of $1 \mathrm{H}$ and $\mathrm{lle}$ at $8 \mathrm{Mb}$, compared with earlier work by Stevenson 23 . 
At $x=0.07$, the He fraction in Jupiter, the demixing temperature is 8000 $\mathrm{OK}$. This estimate of the demixing temperature in Jupiter is open to criticism because the helium atoms in Jupiter from $3 \mathrm{Mb}$ where hydrogen is presumed to pressure ionize on the $35 \mathrm{Mb}$ pressure at the center it is probable that the helium atoms are neutral, i.e. with two bound electrons. Strictly speaking the Hubbard-DeWitt simulation of $\mathrm{H}$ and He applies only to astronomical objects with pressures above 50 to $80 \mathrm{Mb}$ in order to assure the pressure ionization of the He.

The free energy model for the $\mathrm{H}$ - He mixture can probably be considerably improved with more accurate $\mathrm{MC}$ simulations involving up to 1000 charges and up to several million configurations for each value of $\Gamma_{e}$ and $r_{s}$. It should also be mentioned that the ten fitting coefficients used in Eq. 10 can be somewhat reduced in number by using the scaling obtained in recent work by Rosenfeld on charged Yukawa mixtures 24 . Thus the coefficients $a_{3}$ and $a_{4}$ can be replaced by a single coefficient weighted with $\mathrm{Z}_{1}^{7 / 3}$ and $\mathrm{Z}_{2}^{7 / 3}$.

\subsection{Partially Ionized $\mathrm{H}$ and $\mathrm{He}$}

The obvious problem for theoretical calculations of the $\mathrm{H}-\mathrm{He}$ equation of state and the possibility of phase separation of the He in jovian planets is the fact that the hydrogen whether in molecular or atomic form become pressure ionized at approximately $3 \mathrm{Mb}$ while the tightly bound helium electrons remain attached to their nuclei in the $\mathrm{H}$-He mixture until some much larger pressure, $\sim 50 \mathrm{Mb}$. Chabrier and Saumon ${ }^{4}$ have given a detailed model for the equation of state of hydrogen from the molecular region to the fully ionized region that exhibits a plasma phase transition with a critical point at $T_{c}=15300 \mathrm{~K}, P_{c}=0.614 \mathrm{Mb}$, and $\rho_{c}=0.35 \mathrm{gm} / \mathrm{cc}$.

The Chabrier-Saumon EOS is probably the best theoretically based and most quantitative result available for use in modeling the jovian planets. Whether there is a true phase transition or simply a sharply defined region where pressure ionization occurs does not affect the accuracy of the EOS very much. Unfortunately this possible phase transition is out of range of current experimental measurements.

Pure He is believed to be pressure ionized at some far higher pressure, perhaps as much as $80 \mathrm{Mb}$. He atoms mixed in high pressure ionized hydrogen will probably ionize at some lower pressure which may be greater than the interior pressure of Jupiter. To address this problem of the $\mathrm{H}-\mathrm{He}$ mixture in the 10 to $30 \mathrm{Mb}$ range it is essential to have a detailed understanding of the electronic structure of the mixture. Klepeis, Schafer, Barbee, and Ross at Livermore have approached this problem of the 
electronic structure with total energy calculational methods of condensed matter physics at $T=0 \mathrm{~K}$ In this approach the atoms are placed in a lattice, fcc or bcc, and electronic enthalpy, $H(x)=E(x)+P(x)$ V with $x$ as the He fraction, is calculated with the local density approximation. A brief description of the method and band structure results for the $\mathrm{H}-\mathrm{He}$ mixture is given in Ref. 5. A complete report is available from Livermore. The main thrust of this work is an accurate complete $\mathrm{T}=0 \mathrm{~K}$ calculation with the ions on a lattice from which one obtains the enthalpy of mixing from:

$$
\Delta H(x)=H(x)-x H(x=1)-(1-x) H(x=0)
$$

Results for $10.5 \mathrm{Mb}$ are shown in Fig. 2

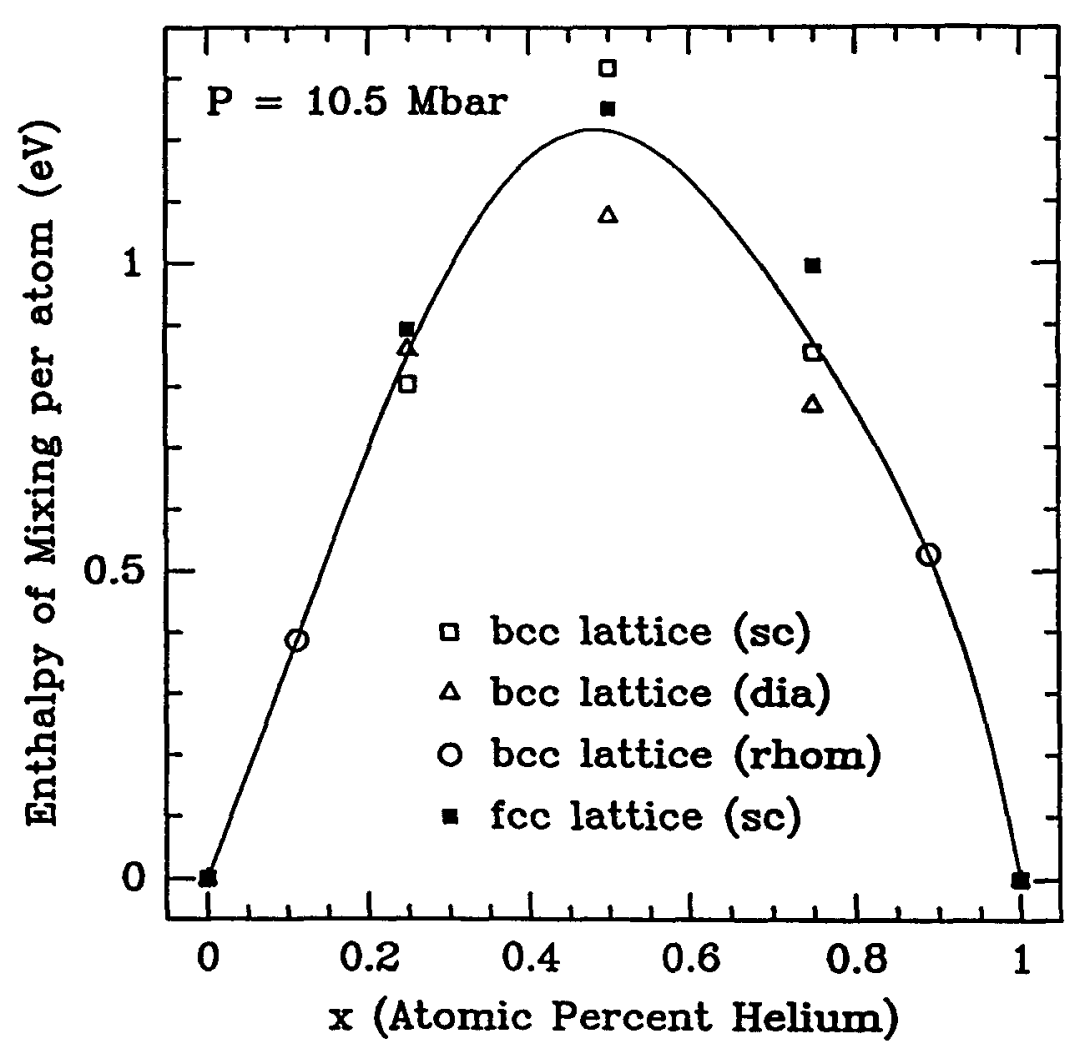

Fig. 2 The enthalpy of mixing per atom in ev from Eq. 13 obtained from first principles total energy calculations for four different lattices at $T=0 \mathrm{~K}$ and $\mathrm{P}=10.5 \mathrm{Mb}$. 
The different lattices used have only a small effect on the mixing enthalpy. Calculations were also done at different pressures to find $\Delta H(x=1 / 2)$. This peak enthalpy was nearly constant between 5 and $20 \mathrm{Mb}$ and dropped only by $35 \%$ at $1000 \mathrm{Mb}$. The large values of the mixing enthalpies are a direct consequence of the fact the helium-derived electrons are more tightly bound to the nucleus than the loosely bound hydrogen-derived electrons.

To go to finite $T$ one needs a Gibbs free energy of mixing. This was done by adding a simple result for the ideal gas mixing entropy to obtain:

$$
\Delta G(x)=\Delta H(x)+k T[x \ln x+(1-x) \ln (1-x)]
$$

and from Eq. 14 the Gibbs mixing free energy was obtained for several temperatures as shown in Fig. 3.

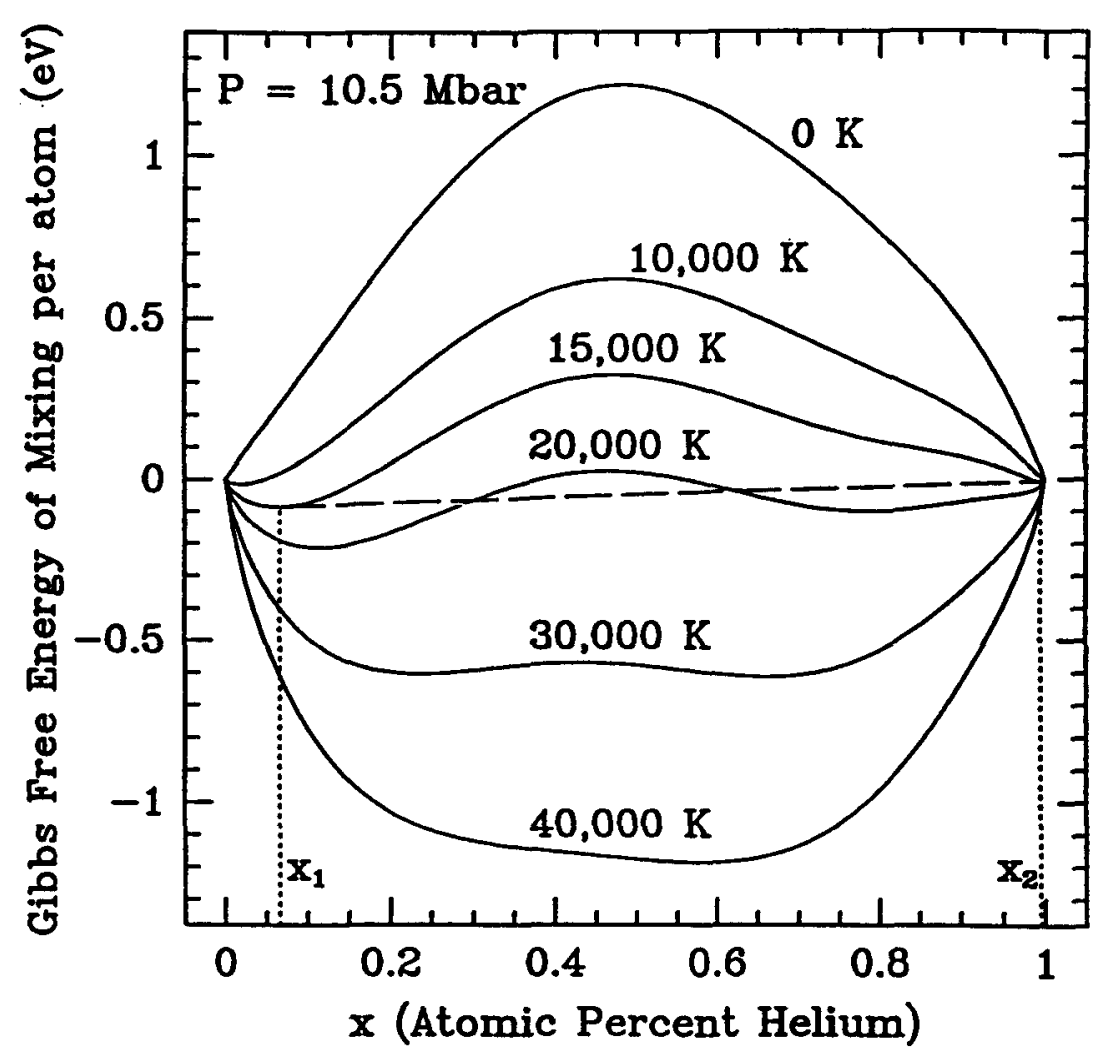

Fig. 3 (iib)s free energy of mixing from Eq. 14 for several temperatures at $10.5 \mathrm{Mb}$. The double tangent construction is shown for $T=15000 \mathrm{~K}$ 
Finally using the double tangent construction at various temperatures the demixing temperature is obtained and shown in Fig. 4

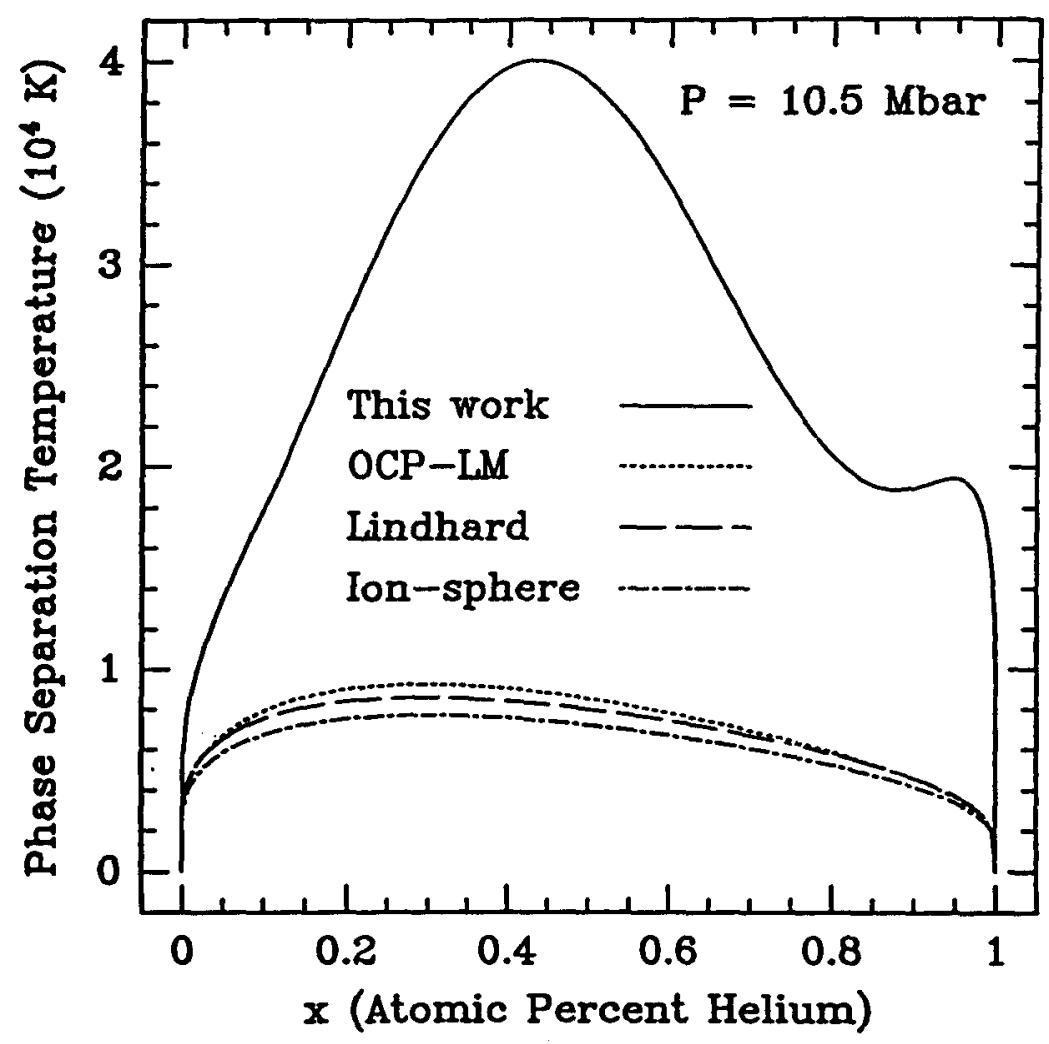

Fig. 4 Immisibility temperature limit for H:He mixtures.

The demixing temperatures obtained by this procedure are very much larger than those obtained by Stevenson and by Hubbard and DeWitt. At $x=0.07$ corresponding to the helium fraction in Jupiter Fig. 4 gives the demixing temperature as close to $15000 \mathrm{~K}$ vs. the $8000 \mathrm{~K}$ from MC with Lindhard dielectric function. The error estimate on this calculation is $\pm 3000 \mathrm{~K}$. However, it is clear that the approach needs serious improvement for finite temperature since $i)$ the ions are assumed to be in a lattice rather than in a fluid state, and ii) the Gibbs thermal energy of the mixture is not included. Clearly more work needs to be done with this approach. The earlier estimate of demixing at $8000 \mathrm{~K}$ is consistent with the belief that $\mathrm{H}$ and $\mathrm{He}$ in Jupiter remain mixed through out much of the planet. If the $15000 \mathrm{~K}$ 
estimate given by the Livermore work is correct, it would have serious astrophysical consequences since it would mean that substantial separation of the He in Jupiter has already happened.

Work performed under the auspices of the U.S. Department of Energy by the Lawrence Livermore National Laboratory under contract number W7405-ENG-48.

\section{References}

1. D.G. Yakovlev and D. A. Shalybkov, Sov. Sci. Rev. E. Astrophys. \&Space Phys. Vol.7, 311-386 (1989)

2. G. Chabrier, N.W. Ashcroft, H.E. DeWitt, Nature 360, 48 (5 Nov. 1992)

3. W.B. Hubbard and H.E. DeWitt, Astrophysical J. 290, 388 (1985)

4. D. Saumon and G. Chabrier, Phys.Rev. A46, 2084 (1992):

Phys.Rev. A44. 5122 (1991)

5. J.E.Klepeis, K.J. Schafer, T.W. Barbee III, M. Ross, Science 254, 986 (15 Nov., 1991)

6. D. Saumon, W.B. Hubbard, G. Chabrier, H.M. VanHorn, Astrophysical J. 391, 327 (1992)

7. G. Chabrier, D. Saumon. W.B. Hubbard, andJ.I. Lunine, Astrophysical J. 391, 317 (1992)

8. D.H.E. Dubin, Phys. Rev. A42, 4972 (1990)

9. H.E. DeWitt, W.L. Slattery, J. Yang, Strongly Coupled Plasma Physics, ed.H.M. Van Horn and S. Ichimaru, University of Rochester Press: Rochester, NY, p.425 (1993)

10. G.S.Stringfellow, H.E. DeWitt, W.L. Slattery, Phys. Rev.A41, $1105(199))$

11. Y. Rosenfeld, Proceedings of this Conference; Phys. Rev. A33, 2025 (1986); Phys. Rev. A37, 3403 (1988)

12. W.L. Slattery, G.D. Doolen, H.E.DeWitt, Phys. Rev.A26, 2255 (1982)

13. S. Ogata,H.Iyetomi,S. Ichimaru, H.M.Van Hom, Strongly Coupled Plasma Physics, ed. H.M. Van Horn and S. Ichimaru, University of Rochester Press: Rochester, NY, p.53 (1993)

14. Y. Rosenfeld, Phys. Rev. A46, 1059 (1992)

15. W.L. Slattery and H.E. DeWitt, recent MC results at Los Alamos

16. S. Ogata, H. Iyetomi, and S. Ichimaru, Astrophys. J. 372, 259 (1991)

17. A. Alastuey and B. Jancovici, Astrophys.J 226,1034 (1978) 
18. J. Iserm,proceedings of this Conference

19. L. Segretain and G. Chabrier, Astron. Astrophys. 271, L13 (1993)

20. G. Chabrier and N.W. Ashcroft, Phys. Rev. A42, 2284 (1990)

21. H. Iyetomi and S. Ichimaru, Phys. Rev. A34, 3203 (1986)

22. W. B. Hubbard and H.E. DeWitt, Astrophys. J. 290, 388 (1985)

23. D.J. Stevenson, phys. Rev. B12, 3999 (1975)

24. Y Rosenfeld, Phys. Rev. E47,2676 (1993)

25. J. E. Klepeis, K.J. Schafer, T. W. Barbee III, M. Ross, Phase

Separation in Mixtures of Hydrogen and Helium at Megabar Pressures, UCRL-JC-107995, July 1991. See also the brief report by the same authors in Strongly Coupled Plasma Physics, ed. H.M. Van Horn and S. Ichimaru, University of Rochester Press:

Rochester,NY, p.73 (1993) 